\title{
Assessing pain in infancy: The caregiver context
}

\author{
R Pillai Riddell PhD CPsych ${ }^{1,2}$, Nicole Racine BSc ${ }^{1}$
}

R Pillai Riddell, N Racine. Assessing pain in infancy: The caregiver context. Pain Res Manage 2009;14(1):27-32.

BACKGROUND: Pain is largely accepted as being influenced by social context. Unlike most other developmental stages throughout the lifespan, infancy is marked by complete dependence on the caregiver. The present paper discusses the primary importance of understanding the caregiver context when assessing infant pain expression.

OBJECTIVES: Based on a review of research from both the infant pain and infant mental health fields, three lines of evidence are presented. First, pain assessment is as subjective as the pain experience itself. Second, assessors must be cognizant of the relationship between infant pain expression, and caregiver sensitivity and emotional displays. Finally, larger systemic factors of the infant (such as caregiver relationship styles, caregiver psychological distress or caregiver acculturative stress) directly impact on infant expression.

CONCLUSIONS: As a result of infants' inability to give a self-report of their pain experience, caregivers play a crucial role in assessing the pain and taking appropriate action to manage it. Caregiver behaviours and predispositions have been shown to have a significant impact on infant pain reactivity and, accordingly, should not be ignored when assessing the infant in pain.

Key Words: Caregiver; Infant pain; Infant pain assessment; Infant pain expression; Parent

$T^{1}$ he importance of caregivers to the healthy development of infants has been asserted for many years (1). A caregiver is not only necessary for meeting the basic needs of an infant, such as feeding and protection, but also for the promotion of higher order needs such as emotion regulation (2). While human infants are born extremely helpless, they are equipped with innate abilities to signal the need for caregiver soothing of their distress. Moreover, similar reciprocal predispositions in the caregiver also help guarantee that caregivers will be receptive to this signalling (3). When healthy infants are in distress, their overall goal is to attain and maintain proximity to a caregiver to obtain a distress-reducing response (4). Infants seek proximity to their caregiver for protection and for external support to regulate their emotions. Because they are preverbal, infants are completely reliant on a caregiver in their immediate environment to be sensitive and recognize their distress, the urgency of their distress signal and to take action to decrease their distress. This need highlights the infant's dependence on

\section{Évaluation de la douleur chez le nourrisson : Contexte de la personne soignante}

HISTORIQUE : On reconnaît généralement que la douleur subit l'influence du contexte social. Contrairement à tous les autres stades du développement et âges de la vie, la petite enfance est caractérisée par une dépendance complète de l'individu envers la personne chargée de ses soins. Le présent article aborde l'importance cruciale de comprendre le contexte de la personne soignante lors de l'évaluation de l'expression de la douleur chez le nourrisson.

OBJECTIFS : À partir d'une synthèse des recherches qui ont porté sur la douleur et la santé mentale infantiles, les auteurs formulent trois théories. Premièrement, l'évaluation de la douleur est aussi subjective que l'expérience de la douleur. Deuxièmement, les évaluateurs doivent connaître le lien entre l'expression de la douleur chez le nourrisson et la sensibilité et les réactions émotionnelles de la personne soignante. Troisièmement, des facteurs systémiques plus généraux propres aux nourrissons (p. ex., son rapport avec la personne soignante, l'état de détresse psychologique ou de stress acculturant de la personne soignante) exercent un impact direct sur l'expression de la douleur qu'ils ressentent. CONCLUSION : Étant donné que les nourrissons ne peuvent pas mettre en mots leur expérience de la douleur, les personnes soignantes jouent un rôle crucial dans l'évaluation de la douleur et dans les mesures appropriées pour la soulager. Il a été démontré que les comportements et les prédispositions des personnes soignantes exercent un impact significatif sur la réactivité du nourrisson à la douleur et, par conséquent, il faut en tenir compte lorsqu'on évalue la douleur chez un nourrisson.

the caregiver and reinforces the caregiver as the most significant context for the young infant.

\section{IMPORTANCE OF THE CAREGIVER IN THE PAIN ASSESSMENT CONTEXT}

Although the importance of the caregiver recognizing and acting to regulate an infant's distress has been well documented in the developmental literature, there has been surprisingly little research examining parental assessment and management of infant pain (3). To understand the infant in pain, an understanding of the dyadic relationship between the infant and the caregiver is necessary (5).

The sociocommunication model of infant pain (6) posits that the infant pain paradigm cannot be fully understood outside the context of the caregiver. Moreover, it suggests that both the infant and caregiver are separately influenced by larger systems such as family, community and culture. An initial read of the model allows one to discern four logical stages that occur

${ }^{1}$ Opportunities to Understand Childhood Hurt Laboratory (OUCH Lab), York University; ${ }^{2}$ Department of Psychiatry Research, Hospital for Sick Children, Toronto, Ontario

Correspondence: Dr R Pillai Riddell, The OUCH Laboratory, Department of Psychology, Faculty of Health, York University, 119 Behavioural Sciences Building, Toronto, Ontario M3J 1P3. Telephone 416-736-2100 ext 33204 or 416-813-6854, fax 416-736-5814,

e-mail rpr@yorku.ca 
after an infant is exposed to a painful event - a painful event triggers the experience of infant pain, the infant transduces the pain experience into an expression of pain, the caregiver assesses the infant's pain expression, and the caregiver intervenes and takes action based on his or her pain assessment. However, although these steps adequately describe the process of a discrete infant pain event, a simple linear chain of events does not capture how a discrete pain event actually occurs within the broader context of an infant and caregiver's past pain-related and nonpain-related interactions. Thus, the model has bidirectional arrows between each of the four stages to portray the complexity of the interaction between the infant and caregiver over time.

The influence of caregiver factors on the assessment of infant pain expression will be the focus of the current discussion. Three lines of reasoning will anchor the discussion: the subjectivity of infant pain assessments, the influence of the reciprocal relationship between infant pain expression and caregiver dispositions (such as sensitivity and emotional displays), and larger parental systemic factors (eg, acculturation, psychological distress and relationship style) that influence infant pain expression.

\section{THE SUBJECTIVITY OF INFANT PAIN ASSESSMENTS}

The International Association for the Study of Pain (7) defines pain as an unpleasant sensory and emotional experience that is always subjective and based on one's past personal injury experiences. This definition implies that the pain experience differs from person to person and is based on several idiosyncratic factors such as past pain experiences, cognitive biases or the person's knowledge base. While the definition does acknowledge that one does not have to be verbal to experience pain, the subjectivity of the pain experience raises two particular challenges for assessing infant pain - the lack of verbal self-report in infancy and the idiosyncratic influences each caregiver brings into the infant pain assessment context, both of which make the accurate assessment of infant pain particularly difficult (3).

\section{Lack of verbal self-report in infancy}

The limited ability of infants to verbally express themselves means that pain assessment in infancy is always relegated to proxy reports of the infant's subjective experience (8). Thus, the infant caregiver is forced to interpret the nonverbal behavioural and physiological changes in the infant to assess pain intensity and the need for intervention. However, previous work has shown that infant pain is definitely in the eye of the beholder. Research with stringent experimental controls on the infant assessment stimuli $(9,10)$ has shown that infant pain assessment is heavily influenced by what caregivers bring into the judgment context (11).

\section{Idiosyncratic caregiver influences on pain assessment}

Idiosyncratic influences that impact empathy and the inclination to help someone in pain were discussed in a literature review conducted by Goubert et al (12). Empathy was described as representing a vehicle through which the adaptive outcome of an observer's behaviour toward the person in pain may be achieved. The authors argued that several variables, unique to the individual, moderate an observer's experience and action toward another person in pain. Some of the variables that were mentioned were cognitive processes such as intentionality, pain experiences and interpersonal judgment. For example, a personal experience with pain was shown to lead to a more readily elicited empathetic response when observing someone in a similar situation (12). This highlights the importance of considering what the assessor (mainly parents, physicians and nurses in the case of infants) is bringing into the judgment context.

The impact of idiosyncratic influences on the perception of another's pain experience is particularly relevant to infant pain assessment. These influences contribute to the subjectivity of infant pain assessments because different caregivers bring different beliefs and experiences to the judgment context. Three analyses $(9,10,13)$, based on a quasiexperimental video judgment study, explored the subjectivity of infant pain assessment by examining immunization pain ratings in different groups of caregivers. Using a $100 \mathrm{~mm}$ visual analogue scale, each participant was asked to judge video clips of the postimmunization pain expressions of infants from five different age groups (two, four, six, 12 and 18 months). Initially, only parental reports of cues important to their pain judgments were examined (9); it was notable that parents did not consider age to be very important. However, when asked how much pain they thought an adult would experience while undergoing the same needle, parents uniformly opined that adults would experience a pain level almost 50\% lower. This finding highlights the challenge of discerning subjective cognitive processes in caregivers because we are dependent on their self-perceptions, which may or may not be reflective of actual practice.

Further analyses of these data $(10,13)$ compared the immunization pain ratings among parents, physicians and nurses. It is important to note that the video stimuli shown to each participant were experimentally controlled - each video clip was coded for pain expression to ensure that a similar level of intense pain was expressed by all infants, regardless of age. Despite every group being shown the same infants with the same level of pain expression, significant differences were found among caregiver groups. Of the three groups, parents attributed the highest pain level and pediatricians attributed the lowest pain level. Nurses' ratings were between the parents and the pediatricians and did not significantly differ from either group. The caregiver differences in judgments were consistent across age groups from two to 18 months of age. The authors suggested that the reason for the differentiation in pain attribution among the groups of caregivers may have been due to factors associated with group membership. For example, parents may have been most sensitive to pain reactions because their assessment experience is primarily based on their own children and they have limited exposure to other children in pain, unlike nurses and pediatricians. However, it was later shown that these higher pain judgments may not translate into better management of infant pain. Despite having significantly lower infant pain judgments than parents, pediatricians had significantly more optimal beliefs regarding the suitability of pain medication for neonates than parents. These types of discrepancies between assessment and management suggest not only the importance of who is doing the judgments but also that different caregivers may not be using the same pain scale the same way. One person's assessment of two of 10 may be 
another person's four of 10 . This may be a clinically significant difference when it comes to potential management options.

As reviewed above $(9,10,13)$, the subjectivity of infant pain assessment has been well documented in healthy infants. More recently, this phenomenon was supported in critically ill infants (14). Elias et al (14) conducted a study to verify whether parents and health professionals homogeneously evaluate the presence and intensity of neonatal pain in a sample of hospitalized infants. In this study, different groups of three adults (consisting of one parent, one nurse's assistant and one pediatrician) evaluated the presence or absence of pain and the intensity of pain for the same infant, during the same 1 min period. The results of the study showed that parents, nurse assistants and pediatricians readily agreed on the absence of pain, indicating that there was homogeneity for when they thought the infant was not experiencing pain. However, when the three groups of adults thought the infants were experiencing pain, they differed with regard to the intensity of pain they perceived the neonates to be experiencing. They found that as the average pain scores for individual infants increased, there was growing disagreement between the observers (ie, there was more disagreement for an infant who received an average pain score of 6 than for an infant who received an average pain score of 2). The authors noted that this was especially concerning because the moderate level is where the decision to administer analgesics is made. There was significant heterogeneity when it came to evaluating neonatal pain intensity. In an attempt to discern the relative influence of infant variables, they found that only a small proportion of the disagreement among observers could be readily attributable to infant factors. This study validated experimental work by finding that even in a naturalistic context where caregivers are standing over the same infant for the same period of time, subjective factors influence infant pain assessment.

The importance of remembering that pain judgments are as subjective as the pain experience is clear through both experimental and clinical studies. Because infant caregivers do not have the luxury of validating their pain assessments against their patients' self-report, it behooves all those involved in the care of pained infants to not only ensure their clinical care algorithms include an evaluation of the factors that may influence the infants' pain expression, but also the contextual factors that may influence their own pain assessments. As posited earlier, the interaction between infant pain expression and caregiver assessment or management is bidirectional. Thus, it is erroneous to simply assume a linear unfolding of events - for example, the infant first expresses pain, then the caregivers' pain care behaviours (assessment and management) are triggered. While it is difficult to debate the elusive influence of parental behaviours on infant pain experience, recent research suggests that such behaviours influence the expression of infant pain.

\section{UNDERSTANDING THE RECIPROCAL RELATIONSHIP BETWEEN INFANT AND PARENT BEHAVIOURS WHEN ASSESSING INFANT PAIN EXPRESSION}

Over time, infants' experiences and expressions of pain are shaped through interactions with their caregivers (15), who may either magnify or moderate the infants' pain-related distress.
Pain is accepted to be an affective and sensory experience and any attempt to untangle the two dimensions is futile. Thus, from a infant development perspective, it would seem to follow that by meeting an infant's basic emotional needs in a painful context (ie, the need to be close and feel secure with a parent during times of perceived danger), the sensory aspect would likely be attenuated. This type of hypothesis is supported by implications of work with older children on the influence of parental anxiety (16).

Research has been examining how parents influence the expression of infant pain through soothing. Thus, in a pain assessment context, it is important to recognize the reciprocal relationship between infant and caregiver. The infant will be sensitive to the caregiver's behaviours, which will influence the infant's pain expression, and impact on the assessment of infant pain. Before the late 1980s, most literature on soothing infants in distress focused on soothing nonpain-related distress, such as prevention of crying, rather than the relief of distress (17). Today, the investigation of the effect of soothing behaviours on pain-related distress is viewed as important to develop effective infant pain interventions. One of the first studies conducted on parental soothing for infants in pain found that levels of crying declined significantly after an injection if swaddling or a pacifier was used to soothe the infant (17). A recent comprehensive review of pain-reducing techniques (18) for immunization pain asserted that parental behaviours observed to decrease infant pain-related distress include the use of parent coping behaviours such as nonprocedural talk or distraction, positive maternal affect and proximal soothing such as rocking, touching, stroking, kissing and maternal vocalizations. Moreover, it has been suggested that the combination of several soothing behaviours, such as holding, rocking and vocalizing, is more effective for soothing infant distress after an inoculation than only using one soothing mechanism (19). In the infant pain context, all of these parental behaviours have been characterized as sensitive. Analyses assumed there was a linear relationship between the amount of parental soothing behaviour and infant pain behaviour (ie, more parent behaviour X will be directly related to more or less of infant behaviour Y). Thus, the majority of studies have focused on specific maternal behaviours and the frequency counts of such behaviours $(19,20)$, as opposed to viewing the mother's behaviours within the context of the infant's reactions. A simple frequency count of maternal behaviours alone can lead to an incomplete analysis of the quality of caregiving because it is widely acknowledged in infant mental health circles that sensitive caregiving relates strongly to following the infant's lead (21). Some infants may find rocking very soothing, while the same behaviour for another infant may actually increase distress. Accordingly, if one were to apply an infant mental health perspective to the infant pain scenario, sensitive caregiving would not be defined from a generalist perspective but rather based on the parameters set by an individual child. This perspective would suggest that different infants may find different parental behaviours soothing and that what behaviours they find soothing may change over time. Following this logic, sensitivity in the pain context should be defined by an infant's positive reaction (ie, lower distress reactivity) to a parental soothing behaviour. Thus, a measure that evaluates soothing behaviours should be one that follows a caregiver's behaviours with an 
ongoing attunement to the impact of those behaviours on the infant.

Taking this particular stance, a study conducted by Din et al (22) investigated the relationship between maternal emotional availability (similar to the concept of maternal sensitivity) and infant pain expressions in an immunization context. Emotional availability is a global way of assessing parental behaviour during an interaction between a caregiver and a child. Emotional availability includes constructs such as parental sensitivity (the ability to 'read' an infant's cues and display appropriate affect while respecting the developmental interests and capabilities of the infant), structuring (the parent's ability to structure the interaction and appropriately meet the needs of the infant), nonintrusiveness (the parent's ability to be available without being intrusive, direct, overstimulating or overpowering) and nonhostility (the parent's ability to avoid abrasive, antagonistic or impatient behaviour). While the measure scores the sensitivity of the mother, as descriptions of the above subscales would suggest, it often does so by examining the infant reaction to maternal behavioural interventions. This ensures that regardless of the actual behaviour (holding, rocking, etc), the influence on the infant is sensitively attempting to be distressreducing. Thus, an infant does not have to be distress-free for the mother to be deemed sensitive; it is about how the mother reacts (and in some cases, continues to react) to the infant's distress. Moreover, by offering a global judgment (as opposed to counting the frequency of maternal behaviours), it allows for some trial and error on the part of the parent and child as they determine what works best for them.

However, a criticism of this measure is that while one is given an overall understanding of whether the caregiver has interacted sensitively or not, we do not know the actual behaviours that contributed to this outcome. This would only be available from more traditional measures that track discrete caregiver behaviours.

Nevertheless, the results revealed that maternal nonintrusiveness was related to lower infant pain expressions immediately and $1 \mathrm{~min}$ following the immunization. Additionally, maternal sensitivity and emotional availability were related to lower infant pain expressions $1 \mathrm{~min}$ after the immunization. These results suggested that intrusive caregiver behaviours (such as trying to dress a child before attempting to soothe his or her distress) can increase infant pain reactivity and thus hinder the regulation of pain-related distress. Alternatively, caregivers who are sensitive and emotionally available help their infants to regulate their pain-related distress. These results support the notion that global judgments of parental sensitivity are important in contextualizing the assessment of infant pain reactivity.

A second study by Horton and Pillai Riddell (submitted manuscript, 2008) further underlines the impact of caregiver behaviours on infant pain expression. This study examined the relationship between mothers' spontaneous facial expressions of pain and fear immediately before their infants received a needle, and infants' facial expressions of pain immediately after the immunizations. The rationale behind this study stemmed from developmental literature emphasizing that infants are drawn to the human face, and maternal affective facial displays predict infant facial displays (23). Based on previous literature, it has also been shown that anxious mothers display reduced maternal sensitivity and reduced emotional tone during interactions with their infants (24). Reduced maternal sensitivity has been further associated with low ability to regulate distress in infants. Based on this literature, it was hypothesized that there would be a positive relationship between maternal fear expression and infant pain expression. However, the results of the study demonstrated a surprising contradiction. In actuality, mothers' facial expressions of fear before the needle were associated with lower levels of infants' pain expression after the needle, indicating that mothers who displayed a mild fear face before the needle had infants who expressed less pain. This was particularly interesting because mothers who expressed more fear before the needle had infants who looked more at the mother before the immunization. Mothers' subtle facial expressions of fear may indicate a relationship history of empathetic caregiving that functions to support infants' abilities to regulate distress following painful procedures. On the surface, these results may appear contradictory, especially considering that maternal anxiety and fear have been previously viewed as maladaptive in the pain context $(16,25)$. However, the authors hypothesized that, given the mild nature of the mother's anxious expression (as opposed to intense), it served as an empathetic and preparatory signal, which gave the infant some warning before the immunization. This study clearly demonstrates that infant pain expression after the immunization is highly dependent on the caregiver's behaviour before the immunization. Therefore, it further supports the proposition that when assessing infant pain, we need to examine infant pain behaviours, as well as the behaviours of caregivers that are present.

\section{DISTAL PARENTAL FACTORS IMPACT INFANT PAIN EXPRESSION}

In addition to parental behaviours being predictive of infant pain expression, it is also important to consider the larger context in which the infant pain context is occurring. Understanding the infant in pain requires a fundamental comprehension of the context in which the pain experience is occurring (6). The sociocommunication model of infant pain theorizes that larger spheres, such as family, community and culture, influence how infant pain is expressed and assessed. However, to date, work examining the influence of these larger spheres is sparse.

A recent study provided the first empirical confirmation, to our knowledge, that maternal variables from domains hypothesized by the sociocommunication model of infant pain significantly predict infant pain variables (11). Using an infant immunization pain paradigm, a sample of 75 mother-infant dyads was videotaped during routine immunizations in a pediatrician's office. No manipulation was conducted because the purpose was to obtain a naturalistic observation of mother and child in the immunization context. Videotape footage allowed for objective coding of validated systems for infant facial expressions of pain and more generalized infant distress behaviours. Before leaving the clinic, mothers were also asked to rate the worst pain they felt their child experienced after the immunization needle.

Then, within two weeks of the immunization visit, mothers were interviewed on the telephone and asked a series of questionnaires that tapped into the constructs of acculturation 
(Vancouver Index of Acculturation [26]; 20 items addressing how much one identifies with their heritage culture and North American culture), psychological distress (Brief Symptom Inventory [27], a general screen of mental health), social support (Multidimensional Scale of Perceived Social Support [28] from significant other, family and friends) and relationship style. Relationship style was determined by a 30-item measure that provides a continuous measure of one's characteristic style in close relationships (29). Each item maps onto one of four relationship subscales (ie, secure, fearful, dismissing and preoccupied) and on completion, a participant receives a score on each of the four relationship styles. Individuals who strongly endorse secure relationships styles have a positive view of themselves and other people and easily build strong relationships with others. A fearful relationship style characterizes individuals who have negative perceptions of themselves and others, and have difficulty maintaining relationships. Individuals who have a negative view of themselves and a positive view of others often endorse a preoccupied relationship style. Lastly, contrary to those with preoccupied relationship styles, individuals who have high levels of self-confidence and negative views of others are characterized as having a dismissive relationship style. These individuals tend to avoid emotional interactions and prefer to rely on themselves. Parents were also asked to recall the amount of pain their infant experienced the day after the immunization. Thus, over the two phases of the study, the analyses examined the various interrelationships among larger maternal spheres of culture, community and family, as well as both objective (behavioural coding of facial expression and general distress behaviours) and subjective (maternal judgments of pain on the day of immunization and maternal recall of pain on the day after immunization) pain measures.

Interestingly, it was found that despite being based on the same infant for the same period of time, the objective and subjective pain measurements were not redundant. When predicting maternal in-clinic pain assessments for the day of immunization using multiple regression techniques, the only variable that accounted for a significant proportion of the variance was the generalized infant distress behaviour measure (38\%). However, when predicting maternal recall of an infant's pain the day after the immunization, infant behaviours in the clinic were irrelevant. The variables that were most predictive of higher infant pain recall were lower identification with North American culture and higher psychological distress. This pattern of findings suggested that when making pain judgments, parents may be most attuned to general infant pain behaviours, but when recalling infant pain, their memories are more significantly influenced by personal factors than infant behaviours. This is an important finding because much of infant health care assessment is based on parental recall of infant symptoms in a doctor's office. Thus, when assessing an infant, health professionals must be aware of how influential parental stressors (such as cultural or psychological stress) are on parent reports in the clinic room.

Underscoring the lack of redundancy between objective and subjective assessments of infant pain and even between objective measures of infant pain, a completely different pattern of results occurred when attempting to predict the objective measures of infant pain. When the global distress behaviour measure was predicted using multiple regression, three variables were significant - the infant's facial expression of pain, the infant's baseline agitation and maternal in-clinic pain assessments. However, when predicting higher infant pain facial expression, both higher global distress reactivity and lower scores on the dismissive relationship style were predictive. An examination of these differences suggests a number of hypotheses regarding systemic factors on infant pain expression. First, global distress behaviours appear to be more influential on maternal pain judgments than the purported infant pain face. Secondly, while global distress was not influenced by mother relationship style, the infant's pain face was. This contrast is quite interesting because the infant pain face was less salient when mothers endorsed the dismissive relationship style. This style is often associated with people who do not like emotional displays in others. Thus, it is noteworthy that global distress behaviours after immunization may be less amenable to contextual influences than the infant pain face. This may be due to the fact that global distress behaviours are more innately hard-wired to 'come on' when faced with distress, while the pain face (often thought to serve the purpose of nonverbally communicating pain to others) may, by its very nature, be more subject to social influences. Finally, the role of baseline agitation differed between the two, suggesting that a priori agitation influences general distress but not necessarily pain expression, perhaps suggestive of an infant's developing ability to discern pain and pain-related distress. However, due to the wide age range of infants in this study, these hypotheses must be tested in a longitudinal sample.

\section{CONCLUSION}

As a result of infants' inability to give a self-report of their pain experience, caregivers play a crucial role in assessing infant pain and taking appropriate action in managing that pain. Caregiver behaviours and predispositions have been shown to have a significant impact on infant pain reactivity and, accordingly, should not be ignored when assessing the infant in pain. Because the multifaceted feedback loops that occur between infant pain experience, infant pain expression, caregiver assessment and caregiver management are continually changing, it is not necessary or even possible to know all the interworkings of the caregiver and infant in a pain context. However, a basic understanding of the role caregivers play in infant pain assessment appears not only attainable, but the basic responsibility of all those who assess infant pain.

ACKNOWLEDGEMENTS: The authors acknowledge the Canadian Institutes of Health Research for supporting the research in the present paper through salary support awards to Dr R Pillai Riddell and operating grant funds. Ms Racine was funded by the Lillian Wright Maternal-Child Health Undergraduate Scholarship. The authors also thank the families who participated in our studies and the clinics who generously supported our lab.

\section{REFERENCES}

1. Bowlby J. Attachment, 2nd edn. Attachment and Loss series, vol. 1. New York: Basic Books, 1969-1980.

2. Kopp CB. Regulation of distress and negative emotions: A developmental view. Dev Psychol 1989;25:343-54.

3. Pillai Riddell RR, Chambers CT. Parenting and pain during infancy. In: Anand KJ, Stevens BJ, McGrath BJ, eds. 
Pain in Neonates and Infants, 3rd edn. Amsterdam: Elsevier, 2007.

4. Ainsworth MD, Blehar MC, Waters E, Wall S. Patterns of attachment: A psychological study of the strange situation. Hillsdale: Erlbaum, 1978.

5. Craig KD, Korol CT, Pillai RR. Challenges of judging pain in vulnerable infants. Clin Perinatol 2002;29:1-13.

6. Craig KD, Pillai Riddell R. Social influences, culture and ethnicity. In: Finley GA, McGrath PJ, eds. The Context of Paediatric Pain: Biology, Family, Society and Culture. Seattle: IASP Press, 2003:159-82.

7. IASP Task Force on Taxonomy. Pain terminology. In: Merskey H, Bogduk N, eds. Classification of Chronic Pain, 2nd edn. Seattle: IASP Press, 1994:209-14.

8. Stevens B, Pillai Riddell RR, Oberlander T, Gibbins S. Assessment of pain in neonates and infants. In: Pain in Neonates and Infants, 3rd edn. Edinburgh: Elsevier Limited, 2007.

9. Pillai Riddell RR, Badali MA, Craig KD. Parental judgments of infant pain: Importance of perceived cognitive abilities, behavioural cues and contextual cues. Pain Res Manage 2004;9:73-80.

10. Pillai Riddell RR, Craig KD. Judgments of infant pain: The impact of caregiver identity and infant age. J Pediatr Psychol 2007;32:501-11.

11. Pillai Riddell RR, Stevens BJ, Cohen LL, Flora DB, Greenberg S. Predicting maternal and behavioural measures of infant pain: The relative contribution of maternal factors. Pain 2007;133:138-49.

12. Goubert L, Craig KD, Vervoort T, et al. Facing others in pain: The effects of empathy. Pain 2005;118:285-8.

13. Pillai Riddell R, Horton R, Hillgrove J, Craig KD. Caregiver beliefs underlying infant pain judgments: Contrasts of parents, nurses and pediatricians. Pain Res Manage 2008;13:489-96.

14. Elias LS, Guinsburg R, Peres CA, Balda RC, Santos AM. Disagreement between parents and health professionals regarding pain intensity in critically ill neonates. J Pediatr (Rio J) 2008;84:35-40.

15. Cramer-Berness LJ. Developing effective distractions for infant immunizations: The progress and challenges. Child Health Care 2007:36;203-17.

16. Chambers CT, Craig KD, Bennett SM. The impact of maternal behavior on children's pain experiences: An experimental analysis. J Pediatr Psychol 2002:27;293-301.
17. Campos RG. Soothing pain-elicited distress in infants with swaddling and pacifiers. Child Dev 1989;60:781-92.

18. Schechter NL, Zempsky WT, Cohen LL, McGrath PJ, McMurtry CM, Bright NS. Pain reduction during pediatric immunizations: Evidence-based review and recommendations. Pediatrics 2007;119:1184-98.

19. Jahromi LB, Putnam SP, Stifter CA. Maternal regulation of infant reactivity from 2 to 6 months. Dev Psychol 2004;40:477-87.

20. Cohen LL, Bernard RS, McClellan CB, MacLaren JE. Assessing medical room behavior during infants' painful medical procedures: The measure of adult and infant soothing and distress (MAISD). Child Health Care 2005;34:81-94.

21. Landy S. Pathways to Competence. Baltimore: Brookes, 2002.

22. Din L, Pillai Riddell R, Gordner S. Maternal emotional availability and infant pain-related distress. J Pediatr Psychol 2009. (In press)

23. Mumme DL, Fernald A, Herrera A. Infants' responses to facial and vocal emotional signals in a social referencing paradigm. Child Dev 1996;67:3219-37.

24. Feldman R, Greenbaum CW, Mayes LC, Erlich SH. Change in mother-infant interactive behavior: Relations to change in the mother, the infant, and the social context. Infant Behav 1997;20:151-63.

25. Jay S, Ozolins M, Elliott CH, Caldwell S. Assessment of children's distress during painful medical procedures. Health Psychol 1983;2:133-47.

26. Ryder AG, Alden LE, Paulhus D, Delroy L. Is acculturation unidimensional or bidimensional? A head-to-head comparison in the prediction of personality, self-identity, and adjustment. J Pers Soc Psychol 2000;79:49-65.

27. Derogatis L. The brief symptom inventory: Administration, scoring and procedures manual, 3rd edn. Minneapolis: National Computer Systems Inc, 1993.

28. Zimet GD, Dahlem NW, Zimet SG, Farley GK. The multidimensional scale of perceived social support. J Pers Assess 1988;55:610-7.

29. Griffin D, Bartholomew K. Models of the self and other: Fundamental dimensions underlying measures of adult attachment. J Pers Soc Psychol 1994;67:430-45. 


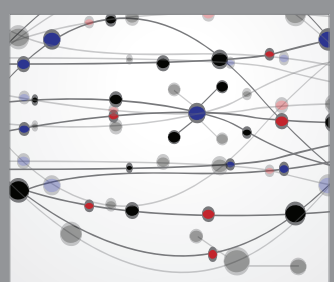

The Scientific World Journal
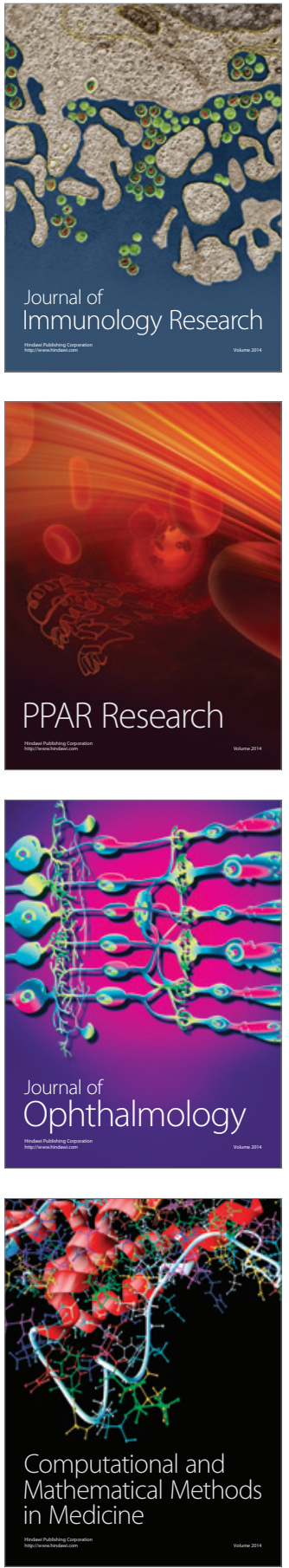

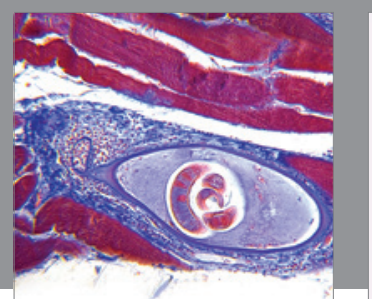

Gastroenterology Research and Practice

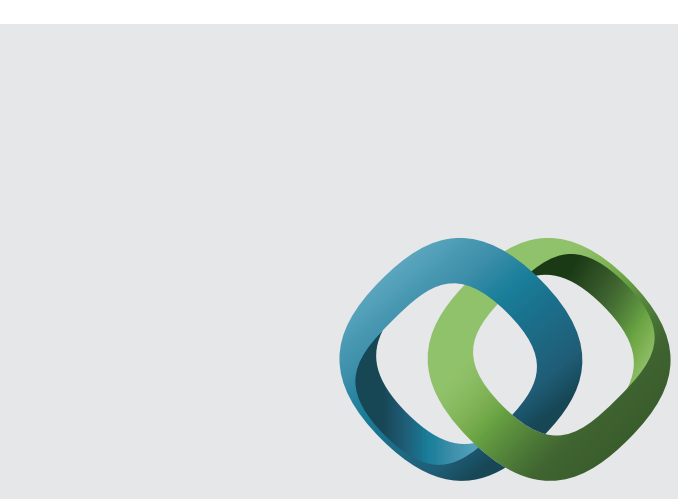

\section{Hindawi}

Submit your manuscripts at

http://www.hindawi.com
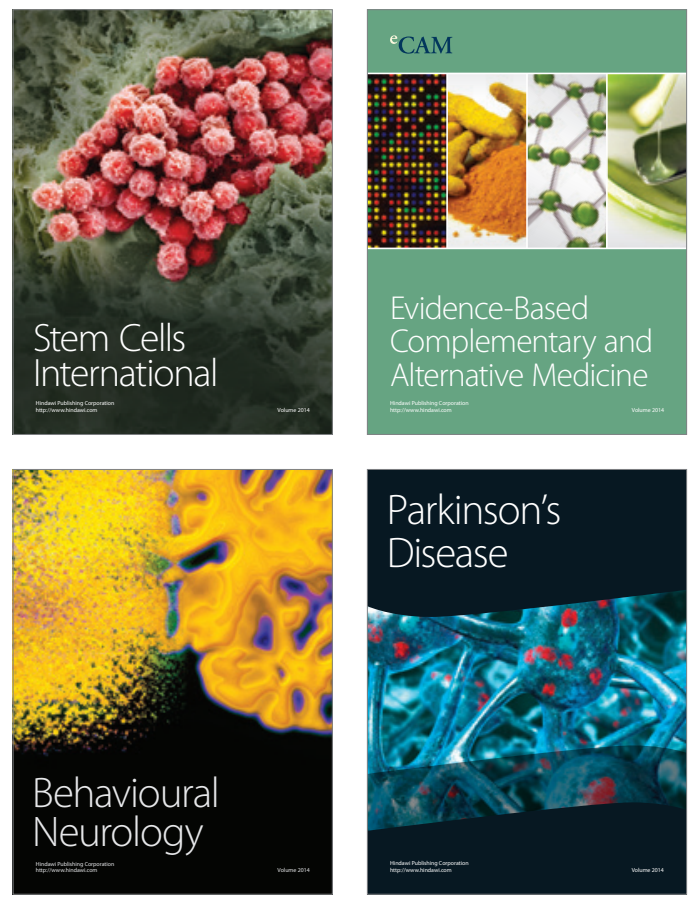
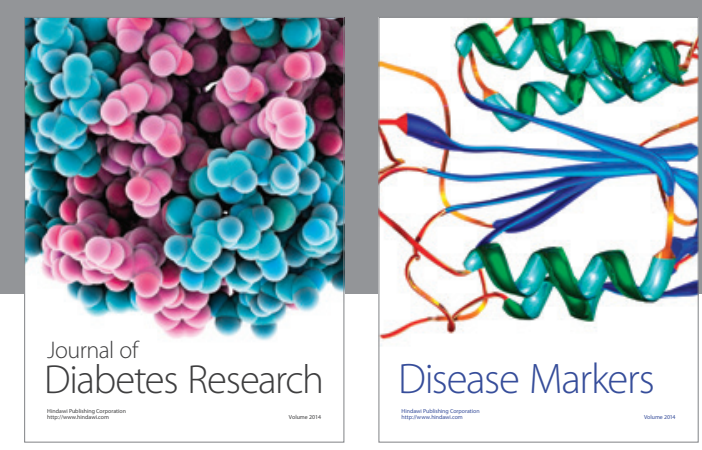

Disease Markers
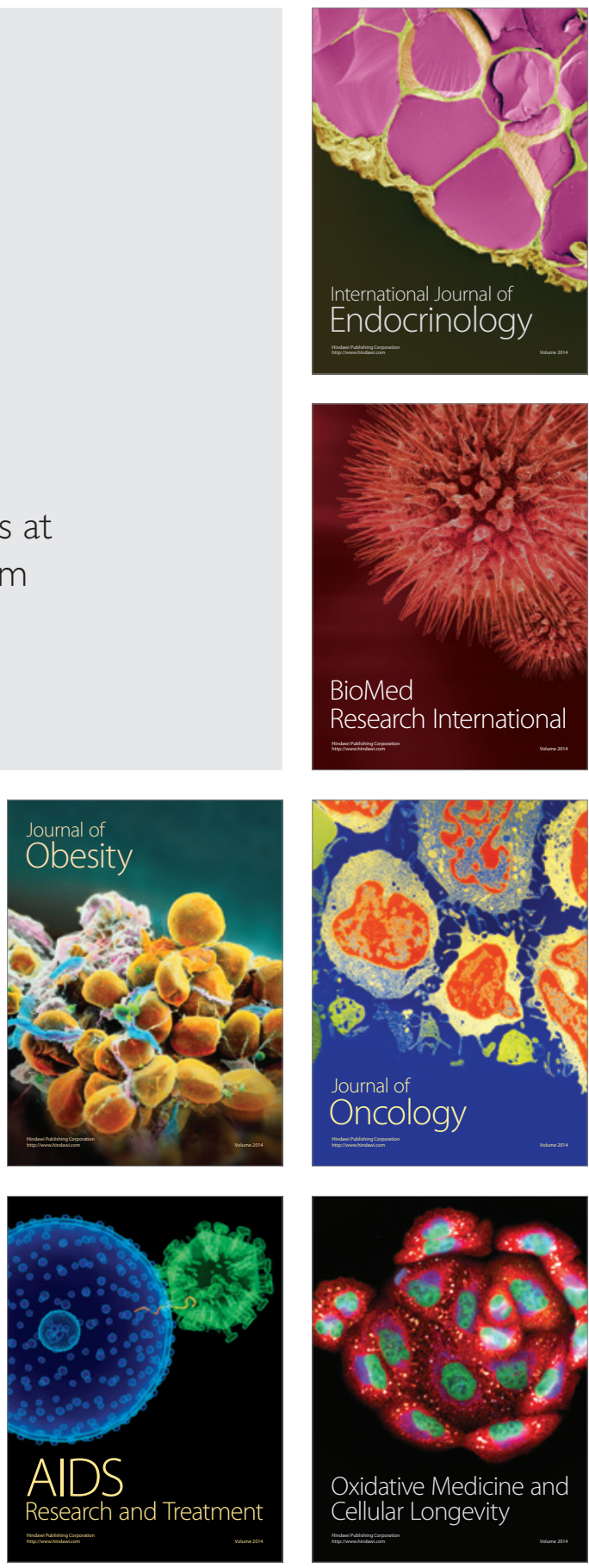